\title{
El mundo de la vida y su relación con las plurales formas de vida en familia ${ }^{1}$
}

\section{The world of life and its relationship with the plural forms of family life ${ }^{1}$}

\author{
Ana María Molina Osorio*
}

\section{Resumen}

El presente artículo tiene como objetivo hacer una cercanía a los trabajos de Alfred Schütz, Peter B. y Thomas Luckmann en Las estructuras del mundo de la vida y La construcción social de la realidad, en relación con la socialización y la construcción de subjetividades e intersubjetividades humanas, a partir de las narrativas escuchadas y percibidas en la voz de actores o sujetos participantes en la investigación "La vida familiar como experiencias subjetiva: cuatro narrativas familiares", que busca explorar, de forma comprensiva, diferentes experiencias de vida en familia, agudizando los sentidos en aquellas acciones emprendedoras que se suscitan de forma diversa para comprender cómo desde de lo subjetivo se puede transformar lo social.

Palabras clave: Mundo de la vida; Sujeto; Subjetividad; Intersubjetividad; Vida en familia.

Candidata a Magister en Educación y Desarrollo Humano, Universidad de Manizales en convenio con el CINDE amolina@tdea.edu.co

Sánchez, A. M., Molina, A. M., Giraldo, C. I, Escobar, G. J., Artículo de la Investigación: "La vida familiar como experiencia subjetiva". Tesis de maestría no publicada para optar al título de magíster en Educación y Desarrollo Humano, convenio CINDE-Universidad de Manizales, Manizales, 2010. 


\section{Abstract}

This article aims to make a closeness to the work of Alfred Schütz, Peter B. and Thomas Luckmann in the structures of the world of life and the social reality, construction on the socialization and the construction of subjectivities and human intersubjetividades, from narratives heard and perceived in the voice of actors or subjects participating in research "family life as subjective"”: four family narratives", which it seeks to explore, in a comprehensive manner, different experiences of family life, sharpening the senses in entrepreneurial activities that will they raise variously to understand how from of the subjective can transform social .

Keywords: World of life; Subject; Subjectivity; Intersubjectivity; Family life.

Con este artículo se busca una aproximación al concepto Mundo de la vida, a partir de las interacciones que se generan en la familia y, desde ella, como experiencia irrepetible y personal, susceptible de ser interpretada, narrada y revivida a través de la expresión y la voz de cada participante que forma parte de encuentros cotidianos y habituales de los que conforman la vida en familia; además, es el lugar en el que se producen sentidos, significados, emociones $\mathrm{y}$ afectos que fortalecen vivencias personales, incomparables e intransferibles.

Se parte del texto Las estructuras del mundo de la vida Schütz (1973) para dimensionar la concepción de mundo de la vida en familia e integrar los conceptos básicos sobre socialización tomados del texto La construcción social de la realidad de Berger y Luckmnn (1968), con el fin de comprender la construcción del sujeto desde lo subjetivo, como lo más personal e intrínseco en la forma de ser, y dar sentido a las vivencias en cada ser humano. Dando relevancia a los procesos de socialización que instituyen al sujeto como aquello que lo acoge y que le brindan elementos para incorporar la realidad social y asumir conciencia frente a aquello que le rodea: a lo cultural, a los diferentes contextos, al mundo social para ejercer acciones sobre él.
Cada sujeto participante de lo social, como ocurre en la familia, se constituye en ella y en las diversas formas de sentir, de interactuar y de dar sentido a las relaciones con los demás; es por ello que Berger y Luckmann son considerados en este trabajo como ineludibles para el análisis del transcurso de la socialización, porque parten de señalar la existencia de dos fases diferentes en ella: una, llamada socialización primaria (que el individuo atraviesa en la niñez convirtiéndolo en miembro de la sociedad), y la otra, de socialización secundaria (referida a cualquier proceso posterior que induce al individuo a incorporar sectores articulares del mundo objetivo de su sociedad), (Berger y Luckmann, 1968). Presentan un acercamiento sociológico que permite interpretar las interacciones cotidianas que se desarrollan entre individuos, construidas en lo social; además, les atribuyen a las interacciones cotidianas ciertas características esenciales, lo que permite superar las reflexiones individuales y centrar el análisis en el modo social en su relación con el mundo con la familia como el primer escenario privilegiado para comentar algunos aspectos de su constitución en relación con la vida cotidiana.

Las contribuciones teóricas obtenidas de Schütz (1968) y Luckmann (1973) y los de 
Husserl (1992) y Weber (1993) en textos como Invitación a la fenomenología y Sobre las teorías de las ciencias sociales, registraron la acción social y el lugar de la familia en contextos sociales, para describir las ideas que producen tales acciones y sus efectos, a partir de relatos y encuentros significativos, que propiciaron conceptos como intersubjetividad, subjetividad, realidad personal, sentido común, entendidos como aquello que confrontan sentires frente a sí mismo y en relación con otros, a asumir consensos preestablecidos que son heredados, a formas de decir y expresar, que hacen parte de un entorno social que permea a la familia; adicionalmente, se instó a los actores a reflexionar y a pensarse como protagonistas en las dinámicas sociales, desde la experiencia inmediata que viven y sienten, primero, como una experiencia del sí mismo y, luego, como experiencia en la que se incluye al otro.

Así mismo, permite comprender la forma de alinear o estructurar el mundo de la vida cotidiana y los acercamientos a la forma como constituye el sujeto en las plurales formas de vida en familia, lo que invita por lo menos a definir algunos conceptos básicos de los autores mencionados, con el objetivo de permitir una mejor percepción de este escrito en conceptos como: realidad, conocimiento, fenomenología y mundo de la vida.

\section{La realidad}

"Es una cualidad propia de los fenómenos que reconocemos como independientes de nuestra propia voluntad" Berger \& Luckmann (1968, p. 13), es decir, todo aquello que nos rodea y que lo consideramos como lo más veraz, lo más evidente; que legitimamos desde la cultura en la que vivimos como algo normal, lógico y de sentido común, y que influye en las formas de pensar, sentir y expresar lo que se vive, como dice el catedrático mexicano, Nolasco Morán Pérez: "La realidad de nuestra vida cotidiana determina en gran medida lo que pensamos, lo que decimos, lo que sentimos y hacemos, esa vida cotidiana nos atrapa y configura nuestro sistema de pensamiento y acciones", es cuando nos movemos de manera "natural" y "normal" en un mundo cultural propio, es el mundo de la cultura heredada, es el primer nivel de interpretación o del sentido común, es el nivel de la doxa (opinión en griego), en el que solemos ubicarnos la mayoría de los mortales, Pérez (2009, p. 1).

\section{El conocimiento}

"Es la certidumbre de que los fenómenos son reales y que poseen características específicas", Berger \& Luckmann (p. 15); se refieren a las experiencias directas que los seres humanos tienen con los hechos y las interpretaciones que hacen esos momentos que se presentan en la vida; es para el sujeto individual y el colectivo una certidumbre de su existencia, de su realidad; es un conocimiento relativo de la cotidianidad que vivimos; es darle estatus de verdadero y comprobado a aquello que conocemos, escuchamos, vivimos y sentimos de forma rutinaria, incluso hasta llegar a considerar que el fenómeno hace presencia ante los sentidos de forma cotidiana como algo normal y natural.

\section{La fenomenología}

En la concepción de lo fenomenológico se reconoce el trabajo de Husserl, quien sirvió de referente para los autores mencionados, y que presenta la fenomenología "como aquella que expresa la intencionalidad de la conciencia: toda conciencia es conciencia de algo y ese algo no es la propia conciencia" Husserl (1992, pp. 35-73); es decir, cuando 
afrontamos la realidad experimentada a partir de lo que se desea, piensa y comprende, empezamos a elaborar reflexiones conscientes $\mathrm{y}$ a interpretarlos en otros contextos y situaciones. Es como si tuviéramos que volver la mirada en situaciones cotidianas y comunes que vivimos y que pueden ser pensadas y reinterpretadas de otra manera para encontrar otros sentidos, otros significados y otras interacciones con el mundo.

\section{El mundo de la vida}

Para comprender mejor las interacciones con el mundo que rodea al ser humano, es importante comprender "el mundo de la vida" según Husserl, quien dice: "El mundo de la vida es lo más conocido para nosotros, lo ya siempre autoevidente en toda vida humana. Hay un conocimiento imperfecto que tenemos aquí, cotidianamente, pero es lo necesario para la praxis cotidiana que todos realizamos" (1992, p. 155). Puede entenderse entonces que todo lo que constituye nuestro modo de pensar y de ser, son las formas de sentir y dar significado a las diferentes situaciones cotidianas; es lo que redirige las formas de hacer análisis de la realidad, de reflexionar y pensar la vida a partir de otros sentidos y significados; es aquello que le da dirección a la forma de actuar y proceder en lo social, lo que recibimos desde la vida en familia y que nombramos como formación humana o espiritual; es lo que se entraña en el ser que hace que uno se identifique en el otro porque eso que dice y expresa nos llega al alma; es el corazón de la subjetividad la que nos permite incorporar de forma natural y clara las formas de pensar y hacer conciencia en el otro. Nos construimos en la diferencia con el otro cuando cultivamos la propia forma de pensar y de ser. En palabras de Cobos: "El mundo de la vida es el mundo del significado, del sentido, aquello que constituye propiamente nuestro cosmos y nos es dado, en primera instancia, como un regalo por nuestros antepasados", Cobos (1999, p. 2).

Este trabajo de investigación concibe la acción de interpretar discursos que dan cuenta de las diversas formas en que se construye la vida familiar, para destacar la reproducción $\mathrm{y}$ transformación de los estilos de vida y de pensamiento. Según esto, y teniendo en cuenta los conceptos mencionados, pueden subrayarse tres bases conceptuales que estructuran el mundo de la vida y que cobran importancia desde lo fenomenológico e interpretativo en el trabajo: la realidad social, el mundo de la vida y la situación biográfica. Cada uno aporta en lo fenomenológico e interpretativo de la siguiente manera:

\section{La realidad social}

Se tuvo en cuenta la realidad social como aquello que rodea a cada familia; la forma como se deja permear por los entornos físicos y materiales; las situaciones culturales $\mathrm{y}$ sociales y las interacciones que allí se suscitan. Cumplieron un papel representativo los objetos y ambientes que cada uno de los integrantes experimentó como suyos, con el que construyó el sentido común para relacionarse con otras personas y los contextos, de acuerdo con las interacciones.

\section{El mundo de la vida cotidiana}

Se trata de la forma en la que cada integrante se comprometió, de manera continua, con modos de ser y de actuar en cada propuesta recibida para narrar su experiencia de vida en familia. Muchas de las intervenciones parecían reguladas en una dinámica familiar, a la vez que parecían estar programadas dentro del ámbito 
de la realidad social que cada uno vivía. Lo que pudo evidenciarse fue la toma de conciencia paraintervenirensusrelatosyasumirposiciones de reflexión que daban idea de modificar lo cotidiano, lo habitual. Esto nos permitió hablar de un "mundo de la vida cotidiana", en el que relataban sus vivencias del día a día con argumentos con nuevas miradas, nuevos sentidos, nuevos sentimientos frente a hechoso situaciones que tocaban su realidad personal.

"El mundo de la vida cotidiana debe entenderse como ese ámbito de realidad que el adulto alerta y que simplemente presupone en la actitud de sentido común. Designando por presuposición todo lo que se experimenta como incuestionable, donde todo estado de cosas es aproblemático hasta nuevo aviso" (Schütz, 1973, p. 25). El concepto de mundo de la vida cotidiana permite resaltar el estado de normalidad incuestionable en el cual las interacciones cotidianas con otros seres humanos, denominados como familia, inscriben en costumbres que entran en estados de naturalidad y rutina en los que el sujeto vive, siente y piensa y que nada perturba; el sujeto se acostumbra a formas y maneras de vivir que, en ocasiones, están marcadas por los antecesores; es más: llegando, incluso, a justificar algunos relatos en la realidad social que le rodea, porque desbordaron la situación biográfica de forma consciente, lo que equivale a decir que se tomaron como propias vivencias de otros y que, de forma intencionada, busca cambiar o transformar esa realidad social.

Al hablar de vida cotidiana en el trabajo realizadoconfamilias, resultanecesarioprecisar que aquello estaba referido a la forma como cada participante actuaba, discernía y pensaba para dirigir su acción. Es menester ratificar que se hablaba desde el mundo compartido con otros, el mundo intersubjetivo en el que es atravesado por la conciencia de quien lo vive, lo protagoniza. Además, pudo evidenciarse que actualizaron recuerdos de interacciones pasadas que expresaban y asumían de forma diversa en cada integrante de la familia, algunos protagonistas de la misma unidad familiar, dejan ver en los gestos, emociones y palabras la propia versión de los hechos como cuando "el padre" de la familia tres, llegaba borracho a casa y generaba reacciones diferentes de rabia, miedo, dolor, tristeza, compañía, camaradería o solidaridad en cada uno de los integrantes, Por ello, en esta aproximación al mundo de la vida desde las narrativas de la vida en familia como experiencia subjetiva, la socialización primaria sigue siendo un marco de referencia para interpretar el mundo posible desde el cual alineanpensamientosindividualesycomunesde la famila, pero, a la vez, permite la reflexión y la comprensión de aquellas percepciones que se alejan de lo común y cotidiando en cada una de ellas, ya que lo subjetivo encuentra sustento en las diferencias de sentir, percibir y significar.

\section{La situación biográfica}

Es considerada como una condición ineludible en cada ser humano, puesto que, según lo argumenta Schütz, "es algo que empieza delimitando toda vida humana en la conjugación de hechos o situaciones que se juegan entre dos límites: unos que pueden ser controlados o se pueden llegar a controlar y aquellos que no dependen del sujeto y están por fuera de su propio control" (Luckmann, 1973, p. 26); es decir, es necesario argumentar la situación biográfica para reconocer el mundo social, que no es independiente y ajeno a los actores de la familia; está sujeto a él de acuerdo con las cargas de significado y sentido que cada uno haya configurado; cada 
uno relata su propia versión de los hechos, cada integrante de la familia relata un mismo evento de múltiples maneras y de acuerdo con sus prácticas sociales, con sus interacciones cotidianas.

La situación biográfica determinó en las familias el modo como se presentaba el escenario de la acción: interpretar lo que allí ocurría y enfrentar sus retos. Ello implicó una relación histórica y, así mismo, adentrarse en la vida mental del otro para conocerlo en su cotidianidad para esclarecer que la ciencia comprensiva e interpretativa en lo social no surge, sino que estudia, lo que más se repite: los hechos, los acontecimientos, las experiencias, las situaciones especiales que aparecen como comunes y que se establecen en el mundo de la vida para mostrar su característica de acción principal en la relación con un ser humano histórico que actúa, interpreta y asume sus realidades.

Para comprender lo fenomenológico en temas como la vida en familia, debe redescubrirse el conocimiento de las cosas mismas en cada entorno, emoción o situación vivida; no se trata de conversar de marcos y referentes conceptuales de familias ni de formalizaciones tradicionales de lo que en ella confluye; más bien, se trata de volver a las cosas mismas, comenzar con las interacciones más sencillas y naturales para eliminar todas las capas de sentido con que la ciencia la ha cubierto. Los participantes de cada situación o vivencia cotidiana ponen su propio sentir en palabras, actos, gestos y emociones, y se dejan atravesar por narraciones conscientes que pasan por la mente y contar sin censuras y prevenciones. Puede decirse, entonces, que el problema de ver la familia como un asunto científico y objetivo se centra en cómo desconectar una de las motivaciones principales que dieron origen a la fenomenología, en el cual el interés de lo subjetivo de un fenómeno se queda en lo objetivo del mismo. Como dice J. San Martín: "El mundo, la realidad, no es sino el conjunto de mis experiencias reales y posibles. No existe un mundo del cual no podamos tener experiencia”, San Martín (1986, pp. 48-49).

Será necesario profundizar la concepción de Husserl sobre sí mismo, en la que la hermenéutica fenomenológica se fundamenta como una ciencia social de la comprensión humana, que permite una aproximación a la vida familiar como "escenario posibilitador de subjetividades”, Husserl (1992, p. 155). Según lo anterior, el ser humano que desee llegar a las cosas mismas de lo que confluye en la familia deberá empezar por reconocer el nivel de la experiencia inmediata dentro de ella; ello permitirá saber que las cosas se deben reconocer no solo en su existencia como tal, sino también en los contenidos, sentires, vivencias y emociones; es decir, que ser parte de una familia -no solo para figurar como integrante de ella- es también padecerla en sus diversidades.

En la investigación pudo vivirse una situación de escucha y observación participante, que no fue ajena a cada encuentro para poder llegar a un saber más instaurado de la vida en familia, debían pensarse los primeros modos de apertura y encuentros de sus integrantes. Se analizaron las experiencias inmediatas de la vida familiar en su doble vertiente: como experiencia constante en la vida de los seres humanos y de la manera en que se ha modificado la mismidad de cada uno de los participantes. Dado lo anterior, se pudo comprender el mundo de la vida familiar del sujeto desde las narrativas y mediante 
la observación de sus actitudes en la forma consciente de percibir, sentir y vivir, para formular interpretaciones y entender su experiencia inmediata en relación consigo mismo y con los demás.

En la familia se construye un conocimiento que según Berger y Luckmann se denomina sentido común, que se presenta como "aquel que se comparte con otros en las rutinas normales y autoevidentes de la vida cotidiana", Berger (1968, p. 39). Es por ello que en las investigaciones comprensivas y sociales sobre lo humano, no se pueden desconocer las interacciones sociales en la relación del sujeto con los demás y, mucho menos, abandonar la forma como el sujeto construye sus subjetividades en los contextos de vida social, y cobran importancia las primeras relaciones que el sujeto mantiene desde su socialización en el hogar y que luego empieza a objetivar cuando llega a contextos externos que educan y formalizan los modos de ser y hacer a través del lenguaje. Así mismo, la sociedad no es ni más ni menos que un conjunto de individuos que comunican o expresan a través del lenguaje (sistema de signos verbales), que es el "sistema de signos de más trascendencia en la sociedad humana”, Berger (1968, p. 85). Las objetivaciones comunes de la vida cotidiana se sustentan por la significación lingüística; por tanto, la comprensión del lenguaje es esencial para cualquier "comprensión de la realidad de la vida cotidiana”, Berger (1968, p. 125), considerando el lenguaje narrado que se expresa mediante gestos y actitudes como un elementos sustanciales en las estructuras del mundo de la vida.

En el trabajo investigativo sobre "Vida familiar como experiencia subjetiva: Cuatro narrativas familiares", se le da valor al lenguaje hablado y al no hablado, asumidos como un sistema de signos en la expresividad humana. El lenguaje expresivo es capaz de identificar a cada individuo porque es cambiante y depende de la cantidad de vivencias y situaciones que se suscitan en la vida familiar, y que van configurándose de acuerdo con la suma de significados y sentires que cada integrante de la familia experimenta y que transmite de una generación a otra, puesto que la capacidad de expresar y de sentir el aquí y el ahora en las interacciones con los demás, contiene cargas de afecto, sentimiento y emociones.

Por medio del lenguaje pueden trascenderse el tiempo y el espacio que separa la propia zona de dominancia de la del otro; puede armonizarse la secuencia propia del tiempo biográfico con la del otro y dialogar con él sobre individuos y colectividades con los que no se está en interacción "cara a cara". En la vida familiar, el lenguaje está acompañado de signos, gestos, palabras y actitudes que establecen distancias $\mathrm{o}$ aproximaciones afectivas entre sus integrantes. Además genera situaciones emocionales cargadas de sentido que no pueden definirse en palabras como amor u odio, indiferencia o perdón. Es necesario escuchar el horizonte de significados que allí confluyen de acuerdo con los interlocutores y su forma de comunicar y que reconstruye realidades cotidianas.

La realidad del mundo hace parte de la realidad social; es más: posee estructuras sociales de muchos significados, en los que cada uno ha nacido y en las que viven y "envejecen" junto con sus semejantes. Además, sustenta de forma clara cómo, a partir de la primera interacción con otros, el niño está incluido en un el mundo posible de sus antecesores, con estructuras de significado (objetivos, medios, 
actitudes) que han sido socialmente delineadas y "presupuestadas".

Schütz sostiene que el individuo tiene capacidad de transformar el mundo que le ofreceen el momento de nacer.Lapreguntaque surge es: ¿cómo puede el sujeto transformar el mundo? Según los autores mencionados y los hallazgos del trabajo de campo con familias, se puede inferir que el individuo puede transformar el mundo preorganizado, como actor social que abandona las tipificaciones o roles asignados porque se ve enfrentado a problemas contrarios a sus intereses, fines, convicciones, o de acuerdo con la conciencia que tenga de ello; y es ahí cuando tiene que encontrar nuevas soluciones y cuestionar lo existente. Por lo tanto, el sujeto crea en ese momento nuevas respuestas y con ellas transforma, en la medida de su acción, los significados, los modos de proceder, las reglas de conductas y además es capaz de reflexionar e interpretar las interacciones normativas.

Uno de los hallazgos teóricos del trabajo con respecto a los autores en mención, se refieren a no desconocer que el actor participante de cada unidad familiar y el pensador social (investigador) en el trabajo sobre "La vida familiar como experiencia subjetiva: Cuatro narrativas familiares", suelen aplicar sus propias recetas y tipificaciones con las que conviven y conectan a otros mundos; relaciones en los que ellos (los otros), son algo más distante, menos personal, es decir, el mundo de los grupos, de las organizaciones sociales que aplican sus propias estrategias de acción.

Para concluir, los individuos reproducen y transforman constantemente la realidad social mediante la interacción intersubjetiva en escenarios como el de la familia, en la que comparte con otros, sentidos y significados diversos, pero que configura las primeras relaciones afectivas, independiente del tipo de vínculo establecido como normal social, y que sale a relucir cuando el sujeto se compenetra conotros contextoshabitualesy queloinvitana construir nuevas cotidianidades sociales.

Se consideró la realidad social de los participantes, su entorno, su relación cotidiana con sus semejantes, dónde viven. Lo que tienen y no tienen; pero siempre vinculados por múltiples interacciones. Cada participante, estableció dialogo desde la situación biográfica, el desarrollado individual de vida, aquello que puede y no puede hacer; así mismo se tuvieron en cuenta las mediaciones culturales que posibilitan encuentros o rupturas con los deseos y decisiones. Lo anterior fue evidenciado en expresiones, acompañados de gestos, sentimientos y sentidos de algunos participantes en cada familia, por ejemplo en la familia cuatro, al escuchar la voz de Jennifer cuando dice: "Uno queda marcado por lo que vive, quiera o no quiera, pues de acuerdo a lo que se vive se actúa" acompaña su relato con gestos de nerviosismo y dolor como si confirmara en el cruce de sus dedos y la unión de sus manos, la comprensión de la situación actual. En la familia dos, La expresión de Iván: "Soy el resultado de lo que viví cuando era pequeño, por esas experiencias soy tímido y en mi caso específico me gusta el trago como le gusto a mi padre", Iván pudo verse nervioso y muy asustado, suda de forma exagerada reconociéndose en su hábito frente al consumo de licor. Estas expresiones permiten escuchar las plurales voces que confirman las interacciones y la realidad social en cada unidad familiar. 
Las familias en este trabajo, se presentan como conjunto de posibilidades para aplicar análisis descriptivo y fenomenológico de la constitución del mundo de la vida cotidiana en los seres humanos organizados alrededor de ella, como una experiencia humana en la que se consideran aspectos como la acción simbólica de lenguajes, sentidos, percepciones y disposiciones culturales, sobre los cuales se fundamenta el mundo de la vida de los participantes comprometidos.

En el trabajo de investigación "La vida familiar como experiencia subjetiva: Narrativa de cuatro familias”, Giraldo, Molina, Escobar, Sánchez (2010)., los actores participantes y las investigadoras se vieron motivados a reescribir sentidos y significados frente al concepto de vida familiar; sin poder tipificar las experiencias que allí se suscitaban y reconocieron diferentes formas de expresar los afectos y de argumentar la vida familiar en lo social, permitiendo resaltar el compromiso, la voluntad y el profundo interés de las familias participantes en la investigación, puesto que compartieron sus historias, intenciones, recuerdos y vivencias más profundas, para reconfigurar las experiencias subjetivas de vida en familia como objetivo central de dicha indagación.

Otro asunto valioso en el encuentro con las familiaseslaincorporacióndeaprendizajesenlo que respecta a la comprensión e interpretación de actitudes, expresiones y signos que marcan lo afectivo y lo emocional en el mundo inmediato(familia), querodeaalindividuoensu cotidianidad, que se mantiene en las relaciones cara a cara y establece vínculos con el otro, en la que un nosotros es el que actúa. Es por ello que una de las mediaciones metodológicas consiste en favorecer la narración y la autobiografía para que se hagan entrevistas conversacionales, relatos autobiográficos y se considere al otro con la posibilidad de ser sentido e interpretado en sus acciones sociales.

El actor o protagonista de cada familia tiene la capacidad de transformar el mundo preorganizado, toda vez que favorezca las comprensiones de su propia experiencia, de su propio sentir en el horizonte de las formas de vida familiar, que generen espacios de reflexión y análisis en los que los significados y sentires argumentando en el otro que también piensa y siente de forma diferente, es decir, transformar y ejercer acciones de cambio, en las que se incorporen otros discursos atravesados por la reflexiones más intimas y personales, en las que se puedan expresar las diferencias. Que se reincorporen nuevas propuestas de diálogo para redescubrir y reencontrar nuevas maneras de comprender el mundo.

\section{Referencias}

Berger,P.\&Luckmann, T.(1968).Laconstrucción social de la realidad. Buenos Aires: Amorrortu Editores.

Cobos, T. L. (1999). Un poco de reflexión al mundo de la vida de Husserl. Tesis de maestría para el programa de Ciencias de la Comunicación, del Tecnológico de Monterrey, México, 1999. Blog de Tania Lu, recuperado el 23 de septiembre del 2009.

Sánchez, A. M., Molina, A. M., Giraldo, C. I, \& Escobar, G. J. (2010). La vida familiar como experiencia subjetiva. Tesis de maestría no publicada para optar al título de magíster en Educación y Desarrollo Humano, convenio CINDE-Universidad de Manizales, Manizales.

Husserl, E. (1992). Invitación a la fenomenología. 
Barcelona: Paidós. (1935/1981). La filosofía en la crisis de la humanidad europea (conferencia de Viena). En Filosofía como ciencia estricta. (135 y ss.). Buenos Aires: Nova.

Pérez, N. M. (2009). Reflexiones y comentarios a algunas ideas planteadas en La construcción social de la realidad de Peter L. Berger y Thomas Luckmann. Recuperado de http:// www.geiuma-oax.net/publicaciones/ Reflexionessobrelaconstrucciondelarealidad. pdf

San Martín, J. (1986). La estructura del método fenomenológico. Madrid: Universidad Nacional de Educación a Distancia.

Schütz, A. \& Luckmann T. (1973). Las estructuras del mundo de la vida. Buenos Aires: Amorrortu.

Weber, M. (1993). Sobre las teorías de las ciencias sociales. Barcelona: Planeta - Agostini. 